\title{
Suppressing Alpha-Hemolysin as Potential Target to Screen of Flavonoids to Combat Bacterial Coinfection
}

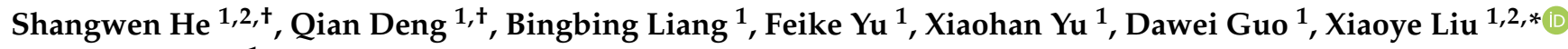 \\ and Hong Dong 1 ,*
}

check for updates

Citation: He, S.; Deng, Q.; Liang, B.; Yu, F.; Yu, X.; Guo, D.; Liu, X.; Dong, H. Suppressing Alpha-Hemolysin as Potential Target to Screen of Flavonoids to Combat Bacterial Coinfection. Molecules 2021, 26, 7577. https: / / doi.org/10.3390/ molecules 26247577

Academic Editors: Daniele Castagnolo, Jürgen Brem, Mark G. Moloney and Sónia Silva

Received: 4 November 2021

Accepted: 12 December 2021

Published: 14 December 2021

Publisher's Note: MDPI stays neutral with regard to jurisdictional claims in published maps and institutional affiliations.

Copyright: (c) 2021 by the authors. Licensee MDPI, Basel, Switzerland. This article is an open access article distributed under the terms and conditions of the Creative Commons Attribution (CC BY) license (https:// creativecommons.org/licenses/by/ $4.0 /)$.
1 Beijing Traditional Chinese Veterinary Engineering Center and Beijing Key Laboratory of Traditional Chinese Veterinary Medicine, Beijing University of Agriculture, No.7 Beinong Road, Changping, Beijing 102206, China; shangwenhe@pku.edu.cn (S.H.); 202030311007@bua.edu.cn (Q.D.); 202030322107@bua.edu.cn (B.L.); 202030322117@bua.edu.cn (F.Y.); yu15512230962@163.com (X.Y.); godv2023@163.com (D.G.)

2 Department of Mechanics and Engineering Science, College of Engineering, Peking University, No.5 Yiheyuan Road, Haidian, Beijing 100871, China

* Correspondence: xiaoyeliu@bua.edu.cn (X.L.); donghongbua@163.com (H.D.)

+ These authors contributed equally to this paper.

\begin{abstract}
The rapid emergence of bacterial coinfection caused by cytosolic bacteria has become a huge threat to public health worldwide. Past efforts have been devoted to discover the broad-spectrum antibiotics, while the emergence of antibiotic resistance encourages the development of antibacterial agents. In essence, bacterial virulence is a factor in antibiotic tolerance. However, the discovery and development of new antibacterial drugs and special antitoxin drugs is much more difficult in the antibiotic resistance era. Herein, we hypothesize that antitoxin hemolytic activity can serve as a screening principle to select antibacterial drugs to combat coinfection from natural products. Being the most abundant natural drug of plant origins, flavonoids were selected to assess the ability of antibacterial coinfections in this paper. Firstly, we note that four flavonoids, namely, baicalin, catechin, kaempferol, and quercetin, have previously exhibited antibacterial abilities. Then, we found that baicalin, kaempferol, and quercetin have better inhibitions of hemolytic activity of Hla than catechin. In addition, kaempferol and quercetin, have therapeutic effectivity for the coinfections of Staphylococcus aureus and Pseudomonas aeruginosa in vitro and in vivo. Finally, our results indicated that kaempferol and quercetin therapied the bacterial coinfection by inhibiting S. aureus $\alpha$-hemolysin (Hla) and reduced the host inflammatory response. These results suggest that antitoxins may play a promising role as a potential target for screening flavonoids to combat bacterial coinfection.
\end{abstract}

Keywords: flavonoids; bacterial coinfections; antitoxin; anti-hemolytic activity; inflammation

\section{Introduction}

The dissemination of bacterial infections accelerates the emergence of antibiotic tolerance [1-3]. However, current antibiotic therapy of bacterial infection leads to post-antibiotic expansion [4], and the resistant bacteria even facilitate the spread of resistance plasmids in the gut to cause multi-bacterial infections [5]. Additionally, the respiratory coinfections correlated with multiple bacterial pathogens are worse than the one with a single kind of bacterium in clinic [6-8]. Meanwhile, the increasing emergence of bacterial coinfections is paralyzing our public health systems worldwide $[9,10]$. Worse still, infections caused by mixed bacteria with unknown mechanisms are diminishing the discovery and development of new antibacterial drugs. Nevertheless, efficacious and novel antimicrobial agents remain the most effective strategy for the treatment of bacterial coinfections. Thus, there are urgent and unmet demands to identify novel or potential targets and to develop new antibacterial drugs with distinct modes of action to prevent such coinfections.

During coinfection, bacterial virulence factors frequently enhance the coinfections, such as where Staphylococcus aureus $\alpha$-hemolysin (Hla) could cause the bacterial coinfections 
by potentiating the opportunistic bacterial infections [11]. In fact, S. aureus Hla belongs to the family of pore forming toxins (PFTs), which not only are involved in bacterial coinfections, but also associated with recurrent infections [11-13]. Therefore, we reasoned that the antitoxin ability of drugs might act as the drug screening principle for controlling bacterial coinfections. The plant natural flavonoids are ubiquitous in medicinal herbs and exhibit various properties on antitoxin and antibacterial activities [14-16]. Thus, flavonoids are utilized to screen drugs of antibacterial coinfections, which may offer alternatively the prospect of more effective therapies against bacterial coinfections through suppressing bacterial virulence factors. Four representative flavonoids including baicalin, catechin, kaempferol, and quercetin were selected to evaluate the therapeutic ability of bacterial coinfections. Among them, baicalin, kaempferol, and quercetin have an antitoxin ability. They target the hemolytic activity of Staphylococcus aureus Hla to combat the coinfection of S. aureus and Pseudomonas aeruginosa. Finally, kaempferol and quercetin indeed have therapeutic effectivity on bacterial coinfections in mouse lung. Since the aim of this paper is committed to finding potential drugs to combat bacterial coinfections, the data have shown that screening of flavonoids though the antihemolytic activity of Hla was feasible. We believe our work will shed light on the development of therapeutic strategies on bacterial coinfections in clinic.

\section{Materials and Methods}

\subsection{Bacterial Strains and Culture Condition}

The bacterial strains including Staphylococcus aureus ATCC29213 and Pseudomonas aeruginosa (a clinical strain isolated from cow dung) were used as the coinfection model strains in this study. Before infection, bacterial strains were cultured in Luria-Bertani (LB) broth (Beijing Aoxing Biotechnology Co., Ltd., Beijing, China) at $37^{\circ} \mathrm{C}$ with agitation (220 rpm). For distinguish of the two strains, Staphylococcus aureus was cultured in mannitol sodium chloride agar (Aobox) and Pseudomonas aeruginosa was grown in pseudomonas Agar Base/CN Agar (Aobox).

\subsection{Flavonoid Treatments and MIC Detection}

Four kinds of flavonoids were employed in this paper. Flavonoids were dissolved in dimethyl sulfoxide (DMSO; Gibco; Shanghai, China) to a final concentration of $100 \mathrm{mg} / \mathrm{mL}$ as a drug storage fluid. More information of Flavonoids was detailed in Table 1.

Table 1. Flavonoids used in this study.

\begin{tabular}{ccccc}
\hline Flavonoids & Molecular Formula & Chinese Herbs & Classic Prescription & Source \\
\hline Baicalin & $\mathrm{C}_{21} \mathrm{H}_{18} \mathrm{O}_{11}$ & Scutellaria baicalensis Georgi & Qingying Decoction & Beijing Solarbio Science \& Technology \\
Catechin & $\mathrm{C}_{15} \mathrm{H}_{14} \mathrm{O}_{6}$ & Paeoniae Radix Rubra & Xijiao Dihuang decoction & Co., Ltd. (Beijing, China) \\
Kaempferol & $\mathrm{C}_{15} \mathrm{H}_{10} \mathrm{O}_{6}$ & Bupleurum chinense & Bupleurum chinense & Shanghai yuanye Bio-Technology Co., \\
Quercetin & $\mathrm{C}_{15} \mathrm{H}_{10} \mathrm{O}_{7}$ & Forsythiae Fructus & Qingying Decoction & Ltd. (Shanghai, China) \\
\hline
\end{tabular}

\subsection{Bacterial Growth Assays}

Bacteria were incubated overnight in $3 \mathrm{~mL}$ of LB broth at $37^{\circ} \mathrm{C}$ with shaking at $220 \mathrm{rpm}$. Then $100 \mu \mathrm{L}$ bacteria $\left(2 \times 10^{6} \mathrm{CFU} / \mathrm{mL}\right)$ were added to a 96-well microtiter plate and treated with another $100 \mu \mathrm{L}$ flavonoids at different concentrations. Finally, bacteria were incubated at $37^{\circ} \mathrm{C}$ and the optical density was measured at $600 \mathrm{~nm}\left(\mathrm{OD}_{600 \mathrm{~nm}}\right)$ for each two hours.

\subsection{Hemolysis Assay}

To evaluate the anti-hemolytic activity of flavonoids, the hemolysis assay was used for detection of the hemolytic activity of $S$. aureus Hla. Briefly, flavonoids were treated with S. aureus for $4 \mathrm{~h}$ and then the supernatants were collected and incubated into $5 \%$ fresh rabbit blood cells for about $30 \mathrm{~min}$ to detect hemolytic activity of the secreted S. aureus Hla. Then, PBS or $0.2 \%$ Triton X-100 were employed as negative or positive controls, respectively. The 
hemolysis rates of flavonoids were detected by using a plate reader (Tecan Infinite 200 pro) and calculating according to previous published work [1,17].

\subsection{ELISA}

The bacterial supernatants were collected to detect the levels of S. aureus Hla by ELISA (Shanghai Jijin Chemistry Technology Co., Ltd., Shanghai, China) according to the instructions.

\subsection{Coinfection of S. aureus and P. aeruginosa In Vitro}

The effect of flavonoids on coinfection of S. aureus and P. aeruginosa were evaluated by using the infected cellular model of pulmonary microvascular endothelial cells (PMVECs) according to the previous method $[18,19]$. Flavonoids including baicalin, catechin, kaempferol, and quercetin (at the concentration of $128 \mu \mathrm{g} / \mathrm{mL}$ ) were used to treat with co-infected PMVECs, which $10^{9}$ cells per well were infected with S. aureus and $P$. aeruginosa at the final concentration ranges from $10^{4}$ to $10^{8}$ colony-forming units (CFUs) in DMEM with $1 \%$ FBS. Finally, the numbers of bacterial mixtures were quantified after $10 \mathrm{~h}$ and the hemolysis of mixtures were detected as per the previous method above.

\subsection{Ethics Statement}

Mice (eight-week mice) were purchased from the academy of military medical sciences, Beijing, China (Certificate Number: SCXK-PLA 2012-0004). All the experiments involved mice were gained an approval by the Institutional Animal Care and Use Committee at the Academy of Military Medical Sciences Institute (Beijing, China; approval no. SYXK2014-0002).

\subsection{Lung Coinfection in Mice}

Eight-week-old male Kunming mice were bred and maintained under specific-pathogenfree conditions. Mice were randomly divided into 9 groups of six each. Mice in the prevention group took $0.5 \%$ CMC-Na or flavonoids $(100 \mathrm{mg} / \mathrm{kg})$ by intragastric administration three days in advance. On the day of challenge, the mice were injected with a $1 \times 10^{9}$ colony-forming units (CFUs) bacterial suspension or saline $(0.05 \mathrm{~mL} / 10 \mathrm{~g})$ through the tail vein. After $2 \mathrm{~h}, 100 \mathrm{mg} / \mathrm{kg}$ of flavonoids or $0.5 \%$ CMC-Na were orally administered to mice in the infected treatment group. After $24 \mathrm{~h}$ infection, the mice were sacrificed by neck removal, and lung samples were collected. Left lungs were fixed in $4 \%$ formalin and submitted for histopathological sectioning and hematoxylin-eosin staining, and the samples were then visualized by light microscopy. Image J was used to analyze the number of inflammatory cells and count the numbers of the pulmonary alveolar area, while the PAA represented the proportion ratio of the pulmonary alveolar area to total area.

\subsection{Western Blot}

Primary pulmonary microvascular endothelial cells (PMVECs) were isolated from 1-day rats as per previous methods $[1,18]$. PMVECs were coinfected with $S$. aureus and $P$. aeruginosa as per the previous method above for $10 \mathrm{~h}$. Then, the co-infected PMVECs were further treated with Hla or treated flavonoids for another $4 \mathrm{~h}$. PMVECs were lysed by RIPA lysis-buffer (Beyotime, Shanghai, China) on ice for $15 \mathrm{~min}$, and then the cell lysates were collected by centrifugation at $10,000 \times g$ for $20 \mathrm{~min}$. The protein concentrations were quantified by a BCA kit (Beyotime, Shanghai, China). Then, proteins $(30 \mu \mathrm{g})$ were separated by SDS-PAGE with $5-12 \%$ polyacrylamide gels and used to analyze the expressions of ASC, caspase-1, and NLRP3. The primary antibodies included rabbit anti-ACS, rabbit anti-caspase-1, and rabbit anti-NLRP3 (Abcam, Shanghai, China) and mouse anti $\beta$-actin (Proteintech, Beijing, China), secondary antibodies were goat anti-rabbit and goat antimouse antibodies (Abcam, Shanghai, China). The protein bands were quantified by ImageJ based on the gray values. 


\subsection{Statistical Analysis}

Statistical analysis was performed using GraphPad Prism 8.0 (GraphPad Software). Data were expressed as the means \pm standard deviations (SD). $p$ values were calculated using unpaired or paired $t$-test between two groups. $p>0.05$ were denoted "ns" and considered as no significant difference. All experiments were performed with at least 3 biological replicates. All animals were used for analysis unless the mice died.

\section{Results}

\subsection{Screening of Flavonoids by the Antihemolytic Activity}

Flavonoid compounds have a basic skeleton with the 2-phenyl-chromone with C6-C3-C6 system (Graphic picture). The antibacterial effects of flavonoids have been previously reported $[20,21]$. Four typical flavonoids including baicalin, catechin, kaempferol, and quercetin (Table 1), which were abundant in plants were selected to assess the direct action to Staphylococcus aureus. Among them, baicalin, which has been shown to have the better effect on bacterial toxins, was used as a reference to screen flavonoids [22]. Results showed that all the four flavonoids have antibacterial activities (Figure 1A), especially in the $8 \mathrm{~h}$ treatment (Figure 1B). Subsequently, the antihemolytic activity of flavonoids against $S$. aureus Hla were detected (Figure 2A); results showed that catechin did not have any antihemolytic activity under the concentrations of $8-128 \mu \mathrm{g} / \mathrm{mL}$, whereas baicalin, quercetin, and kaempferol could inhibit the hemolysis of Hla (Figure 2B,C). In addition, these flavonoids exhibited different levels of antihemolytic activity, namely, the effective concentration of kaempferol began at $8 \mu \mathrm{g} / \mathrm{mL}$ treatment, quercetin $32 \mu \mathrm{g} / \mathrm{mL}$, and baicalin at $128 \mu \mathrm{g} / \mathrm{mL}$ (Figure 2C). Further, to verify whether the flavonoids inhibited the hemolysis of Hla, depending on directly targeting Hla secretion from S. aureus, we quantified the amount of Hla by ELISA. As shown in Figure 2D, the similar tends of Hla levels under the treatment of flavonoids were detected compared to the antihemolytic activity. Altogether, our results suggested that the antihemolytic activity of flavonoids dominated the inhibition of Hla levels. Based on this screening principle, we selected two flavonoids included kaempferol and quercetin as having the best efficacy.
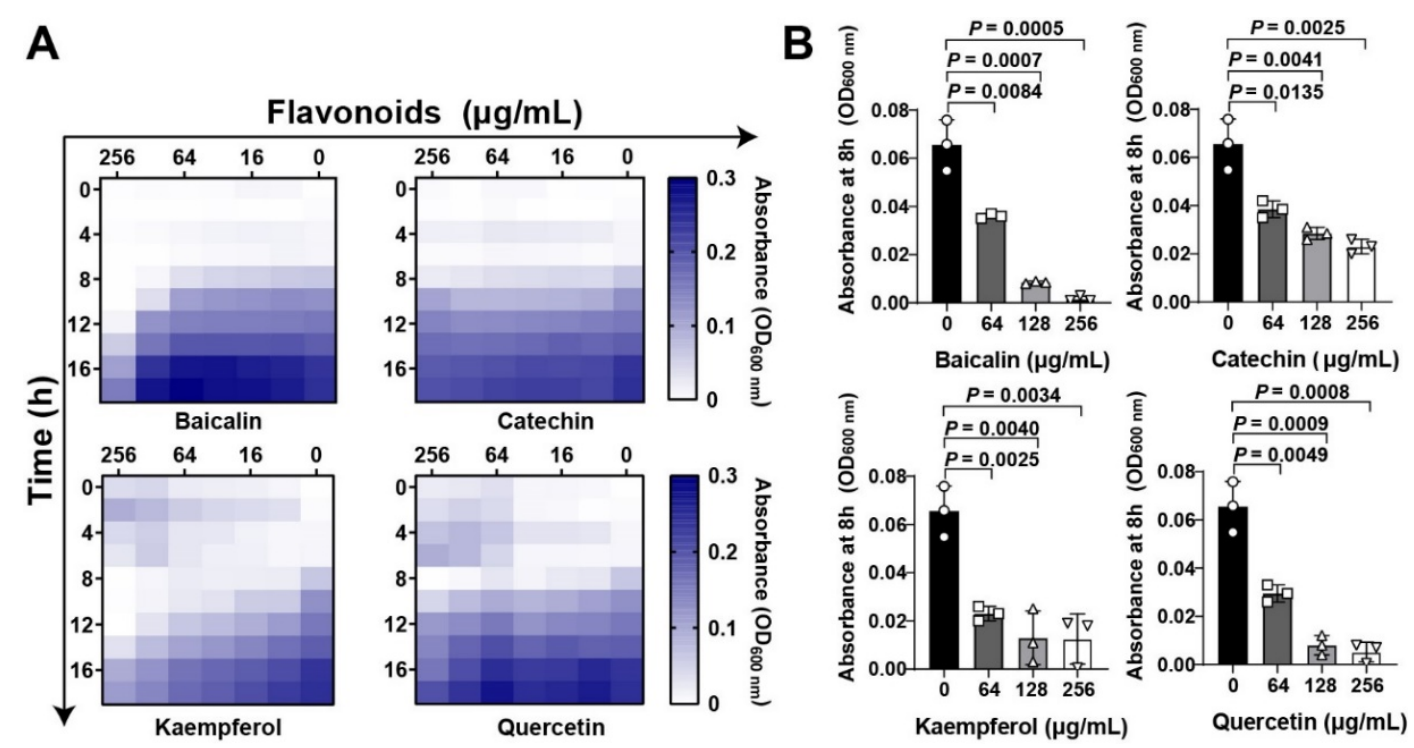

Figure 1. Flavonoids inhibit Staphylococcus aureus growth at the initial period: (A) Flavonoids inhibited S. aureus in both time- and dose dependent manners. Chessboard method was employed to detect the antibacterial effect of flavonoids. Different concentrations of flavonoids including baicalin, catechin, kaempferol, and quercetin (0 to $2048 \mu \mathrm{g} / \mathrm{mL}) \mathrm{were}$ treated with $S$. aureus at different time points $(0,4,8,12,16$, and $18 \mathrm{~h})$. Bacterial growth was quantified based on the absorbance by a plate reader at wavelength of $600 \mathrm{~nm}$. (B) Quantification of $S$. aureus absorbance at $8 \mathrm{~h}$ under flavonoid treatment at the final concentrations of $0,64,128$, and $256 \mu \mathrm{g} / \mathrm{mL}$. Data were shown as Mean $\pm \operatorname{SED}(\mathrm{n}=6)$. 
A

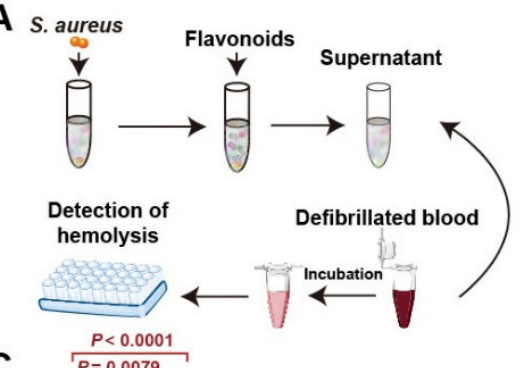

C $\quad \frac{P<0.00079}{P=0.079}$
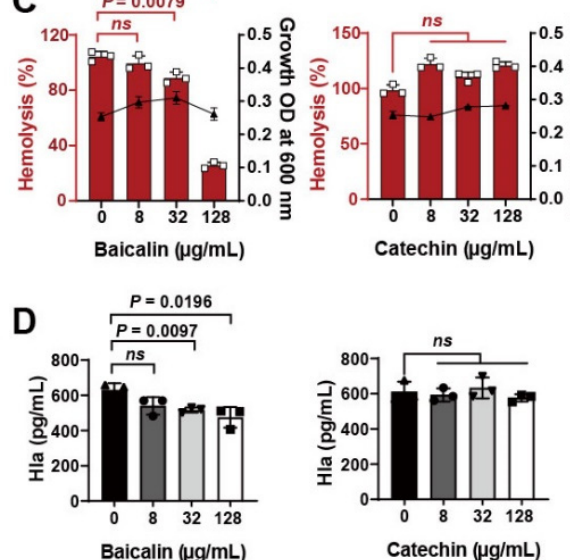

B
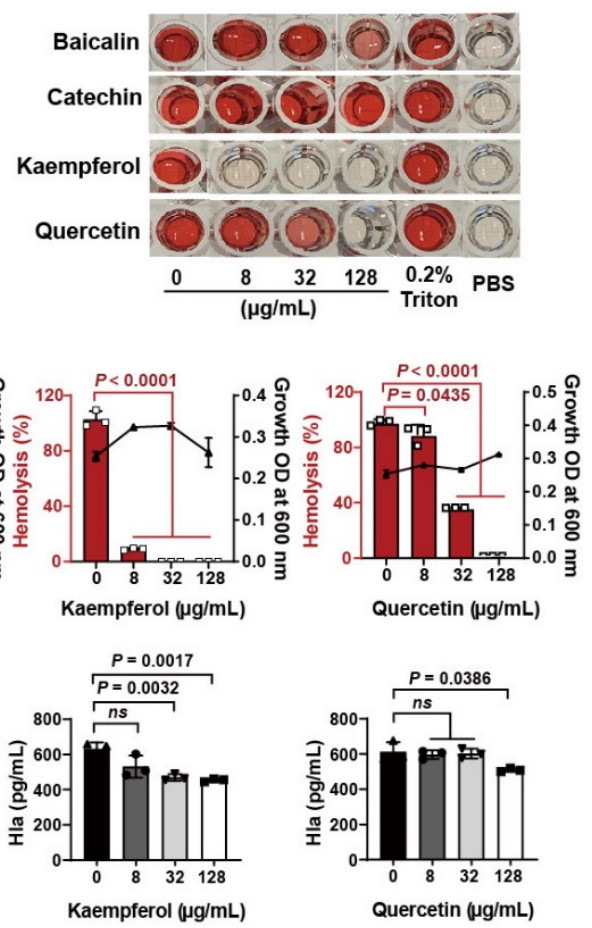

Figure 2. Screening of flavonoids on the hemolytic activity of S. aureus Hla: (A) Detection of hemolytic activity workflow. (B) Images of flavonoids treated with S. aureus Hla in blood cell suspension. The supernatants of S. aureus treated with different concentrations of flavonoids $(0-128 \mu \mathrm{g} / \mathrm{mL})$ incubated with the rabbit blood cell suspension for $20 \mathrm{~min}$. (C) Quantification of hemolytic activation of flavonoids. (D) Detection of Hla levels by ELISA. Experiments were performed with at least 3 independent repeats. $n s$ indicates $p>0.05$ showed no significant differences.

\subsection{Flavonoids Combat the Coinfection of Staphylococcus aureus and Pseudomonas aeruginosa by Targeting Hla}

To assess whether the flavonoids impacted on bacterial expansion in vitro. Firstly, bacterial suspensions of $S$. aureus and Pseudomonas aeruginosa were infected with pulmonary microvascular endothelial cells (PMVECs) in different proportions from $10^{4}$ to $10^{8} \mathrm{CFU} / \mathrm{mL}$, results suggesting that kaempferol and quercetin have better inhibitions of bacterial coinfections (Figure 3A). Then, the bacterial supernatants were collected to determine the antihemolytic activity (Figure 3B). Date showed that baicalin, kaempferol, and quercetin significantly reduced the levels of Hla, revealing that flavonoids inhibited the growth of mixed bacteria and might be regulated by suppressing Hla (Figure 3C). Overall, these findings confirming that inhibition of Hla can decrease bacterial coinfections.

To further investigate the therapeutic efficacy of flavonoids against bacterial coinfections in vivo, a lung infective model was employed to detect (Figure 4A). Briefly, mice were infected with $10^{9} \mathrm{CFUs}$ of $S$. aureus and P. aeruginosa. Then, mice were pretreated with flavonoids for 3 days and then co-infected with bacteria, or the co-infected mice were treated with flavonoids after $2 \mathrm{~h}$ coinfection. Finally, pathological and antibacterial analysis was carried out at 5 days for pretreated groups and $24 \mathrm{~h}$ for treated groups. The quantification of bacteria in lung tissues showed that kaempferol has a more curative effect than quercetin either in pretreatment or treatment of flavonoids (Table 2, Figure 4B,C). This is consistent with the results of antihemolytic activity between the two flavonoids, which showed that kaempferol was also superior to quercetin (Figure 2C). It is worth noting that the bacterial loading of $S$. aureus in mouse lung from the other groups showed no significant changes compared to the untreated group, only the numbers of $P$. aeruginosa from coinfected mice were decreased under kaempferol pretreatment or treatment (Figure 4B,C), probably due to down-regulation effect of kaempferol on Hla levels of $S$. aureus to reduce 
dissemination of P. aeruginosa. Altogether, these data suggest that the better inhibition of bacterial virulence factor, the better anti-coinfection of flavonoids.

A

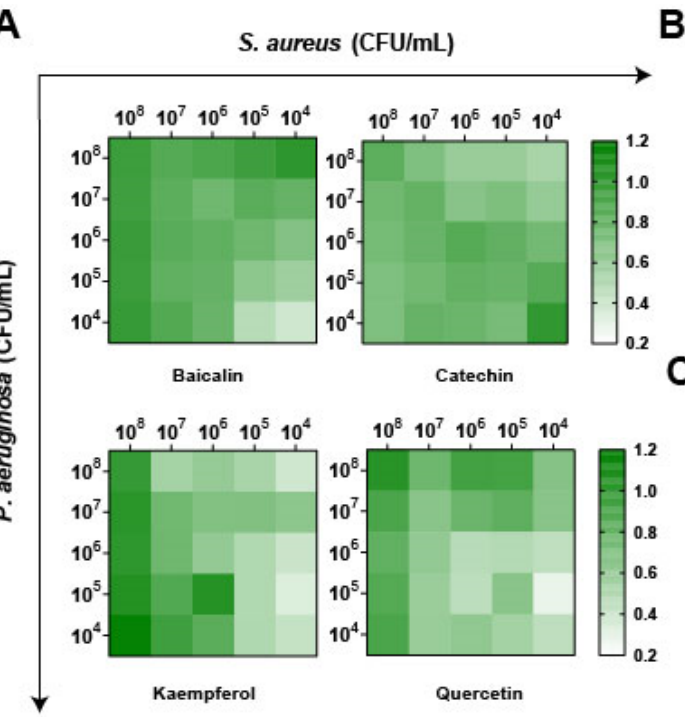

B Untreated Baicalin Catechin Kaempferol Quercetin
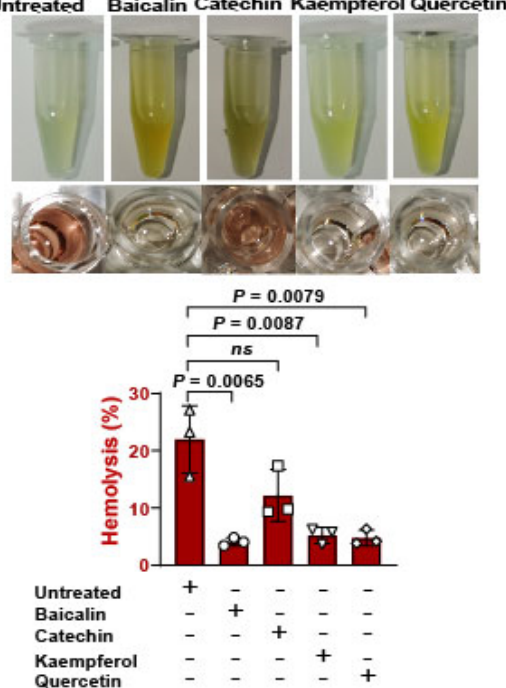

Figure 3. Detection of flavonoids on the growth of S. aureus and P. aeruginosa in vitro: (A) The antibacterial function of flavonoids against the coinfection of $S$. aureus and P. aeruginosa. Pulmonary microvascular endothelial cells (PMVECs) were infected with the mixture of $S$. aureus and P. aeruginosa at different CFUs $\left(10^{4}\right.$ to $\left.10^{8} \mathrm{CFU} / \mathrm{mL}\right)$ and treated with flavonoids including baicalin, catechin, kaempferol, and quercetin (at the concentration of $128 \mu \mathrm{g} / \mathrm{mL}$ ) for $10 \mathrm{~h}$. (B) Images of the hemolytic activity of bacterial mixtures of $S$. aureus $\left(1 \times 10^{8} \mathrm{CFUs}\right)$ and P. aeruginosa $\left(1 \times 10^{8} \mathrm{CFUs}\right)$ treated with flavonoids for $10 \mathrm{~h}$. (C) The hemolysis detections of the mixtures of S. aureus and P. aeruginosa after the flavonoid treatment.
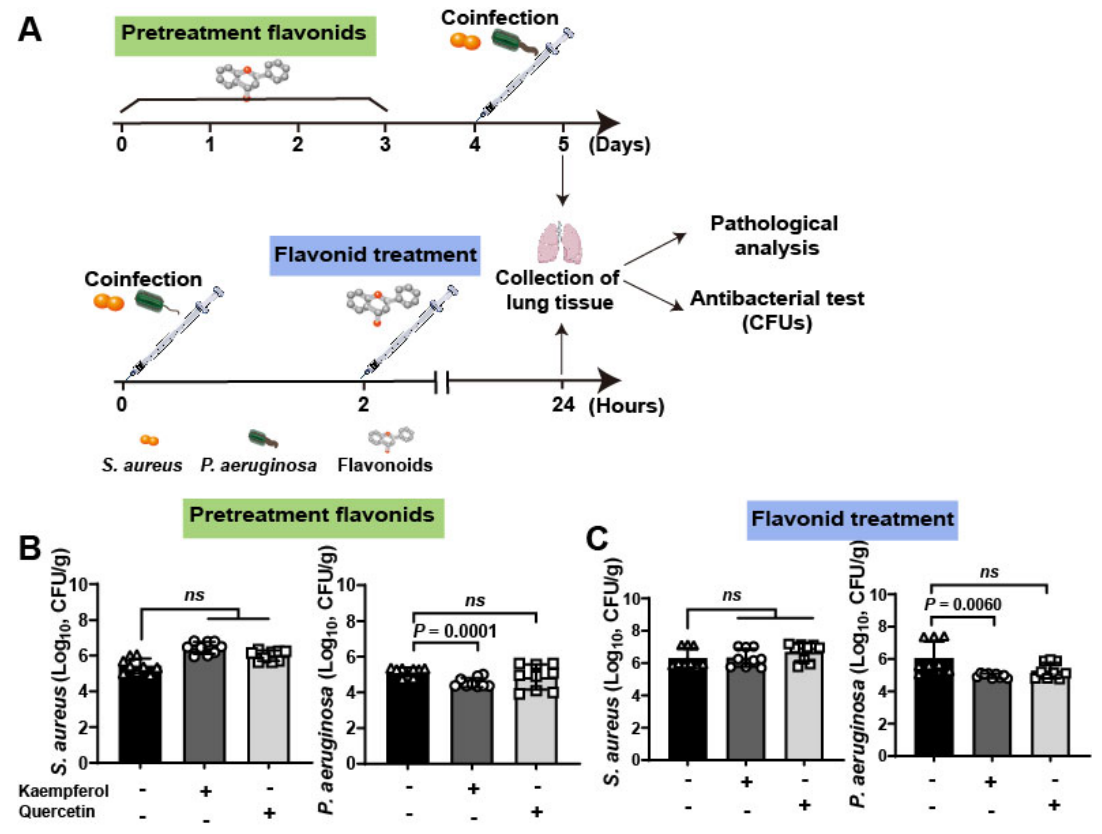

Figure 4. Clinical validation of flavonoids on the mouse lung coinfection of S. aureus and P. aeruginosa: (A) Scheme of the pre-treatment and treatment of flavonoids on mouse lung coinfection. Briefly, mice were coinfected with $S$. aureus $\left(1 \times 10^{9}\right.$ CFUs $)$ and $P$. aeruginosa $\left(1 \times 10^{9}\right.$ CFUs $)$ and flavonoids were pre-treated or treated with infected mice for further determine the therapeutic effects. (B,C) Kaempferol had the better antibacterial effects on the lung coinfection of $S$. aureus and $P$. aeruginosa compared to quercetin on both pre-treatment (B) and treatment $(\mathbf{C})$. 
Table 2. Flavonoid treatment had a significant effect on body weight but no effect on organ index in co-infected mice.

\begin{tabular}{|c|c|c|c|c|c|c|}
\hline \multirow{2}{*}{ Group } & \multirow{2}{*}{ Weight (g) } & \multicolumn{5}{|c|}{ Index $(\%)$} \\
\hline & & Heart & Liver & Spleen & Lung & Kidney \\
\hline Saline solution & $1.42 \pm 0.60$ & $0.78 \pm 0.21$ & $5.75 \pm 0.30$ & $0.44 \pm 0.03$ & $0.66 \pm 0.12$ & $1.48 \pm 0.11$ \\
\hline S. aureus & $4.64 \pm 1.94 \# \#$ & $0.69 \pm 0.12$ & $5.74 \pm 0.44$ & $0.58 \pm 0.09$ & $0.78 \pm 0.06$ & $1.54 \pm 0.06$ \\
\hline P. aeruginosa & $3.67 \pm 2.27^{\#}$ & $0.82 \pm 0.10$ & $6.44 \pm 0.57$ & $0.64 \pm 0.06^{\# \#}$ & $0.75 \pm 0.07$ & $1.70 \pm 0.15$ \\
\hline Coinfection & $6.02 \pm 0.85^{\# \#}$ & $0.69 \pm 0.11$ & $6.10 \pm 0.49$ & $0.47 \pm 0.06$ & $0.75 \pm 0.02$ & $1.78 \pm 0.19$ \\
\hline Coinfection with Kaempferol treatment & $3.77 \pm 1.96^{*}$ & $0.63 \pm 0.28$ & $6.64 \pm 1.02$ & $0.47 \pm 0.07$ & $0.76 \pm 0.03$ & $1.63 \pm 0.17$ \\
\hline Coinfection with Quercetin treatment & $3.81 \pm 1.18^{* *}$ & $0.67 \pm 0.10$ & $6.59 \pm 0.45$ & $0.47 \pm 0.04$ & $0.79 \pm 0.07$ & $1.73 \pm 0.10$ \\
\hline
\end{tabular}

Note: ${ }^{\#} p<0.05,{ }^{\# \#} p<0.01,{ }^{\# \#} p<0.001$ compared with the uninfected group. ${ }^{*} p<0.05,{ }^{* *} p<0.01$ compared with the co-infection group ( $\mathrm{n}=6$ ).

\subsection{Flavonoids Therapy the Bacterial Coinfection Require to Reduce the Host Inflammatory Response to Assist}

To investigate the treatment differences between kaempferol and quercetin on combating bacterial coinfection, the pathological detection of co-infected lung tissues was analyzed by H\&E staining. Images showed that the increased inflammatory cells (black arrowheads) accompanied with congestion points (blue arrowheads) in the lung tissue were only found from co-infected mice compared to $S$. aureus or P. aeruginosa alone infections (Figure 5A(upper),B). In addition, the P. aeruginosa infection and bacterial coinfection decrease the pulmonary alveolar area (PAA), suggesting that damage of lung tissue occurred (Figure 5C). Additionally, pretreatment or treatment of flavonoids with co-infected mice showed that kaempferol and quercetin had the pretreated function for bacterial coinfection, while only kaempferol could treat the co-infected mice (Figure 5A,D,E). These findings indicate that flavonoids decrease the tissue damage, and inflammation might have assisted the therapy of bacterial coinfection.

A

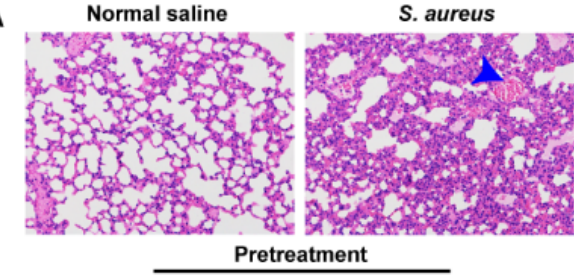

Kaempferol
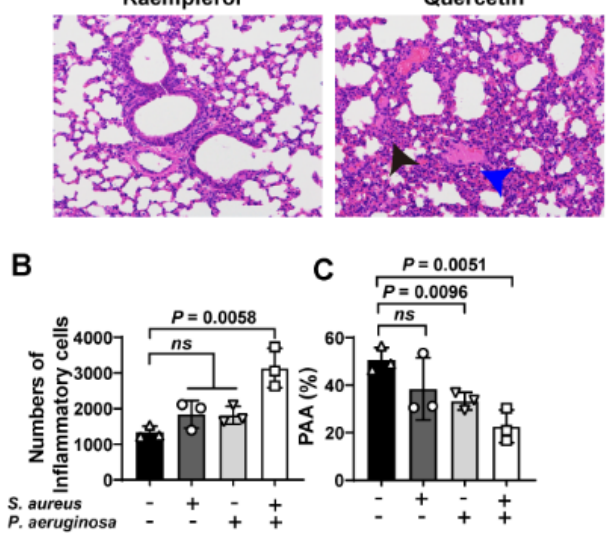

P. aeruginosa

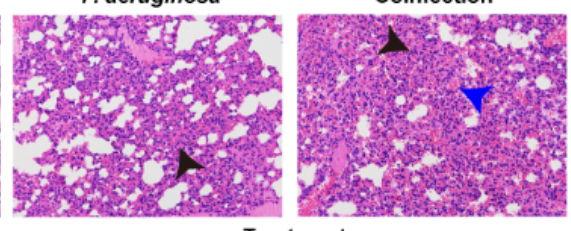

Treatment

Kaempferol Quercetin
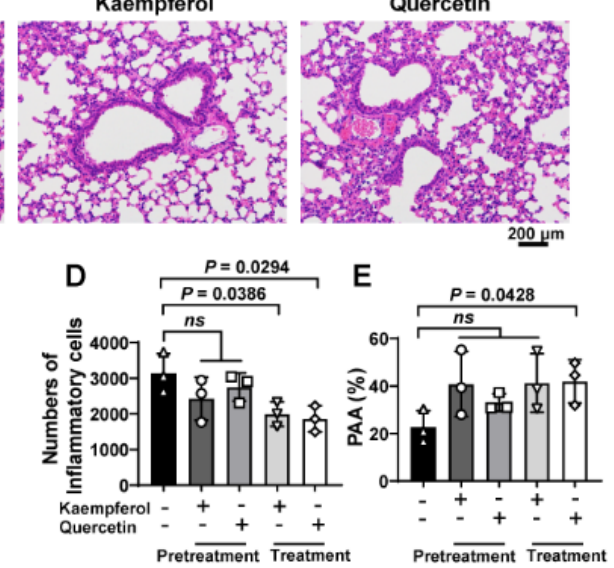

Figure 5. Flavonoids have a better therapeutic effect on lung coinfections of S. aureus and P. aeruginosa in vivo: (A) H\&E staining of the lung tissue. Mice were infected with S. aureus, P. aeruginosa or the mixture of S. aureus and P. aeruginosa. Then, infected mice were treated with flavonoids. The black arrows show inflammatory cell infiltration. The blue arrows indicate the structure of the alveolar cavity. Scar bar $=200 \mu \mathrm{m}$. (B-E) Quantitative analysis of inflammatory cells number and pulmonary alveolar area (PAA) in mouse lung tissues. (B,C) Quantification of the number of inflammatory cells and the PAA in the lung tissues of infected mice. (D,E) Quantitative analysis of pulmonary inflammatory cells and PAA after prevention or treatment with kaempferol or quercetin. Three independent experiments were performed to obtain stable results. $n s$ indicated no significant difference when compared with the non-treated controls. 
Previously, we had confirmed that flavonoids therapied the bacterial coinfection though suppressing Hla (Figure 3). Thus, we further investigate the impact of flavonoids on inflammatory pathway of the Hla-treated PMVECs. Firstly, we found that Hla indeed enhanced the inflammation of co-infected PMVECs by upregulations of the inflammatory proteins of ASC, caspase-1, and NLRP3 (Figure 6A,B), which might explain why the coinfected mice had larger numbers of inflammatory cells (Figure 5A,B). Next, kaempferol and quercetin significantly reduced the inflammation of the co-infected PMVECs in a dosedependent manner (Figure 6A,B), suggesting that inhibition of the inflammation was required in the antiinfection strategy of flavonoids. Lastly, we further detected the inflammatory factors of IL-1 $\beta$ and IL-18; results also revealed that kaempferol and quercetin could decrease the inflammation (Figure 6C). Taken together, our results illustrate that to what extent the flavonoids therapied the bacterial coinfection, depended on the decreased inflammation.

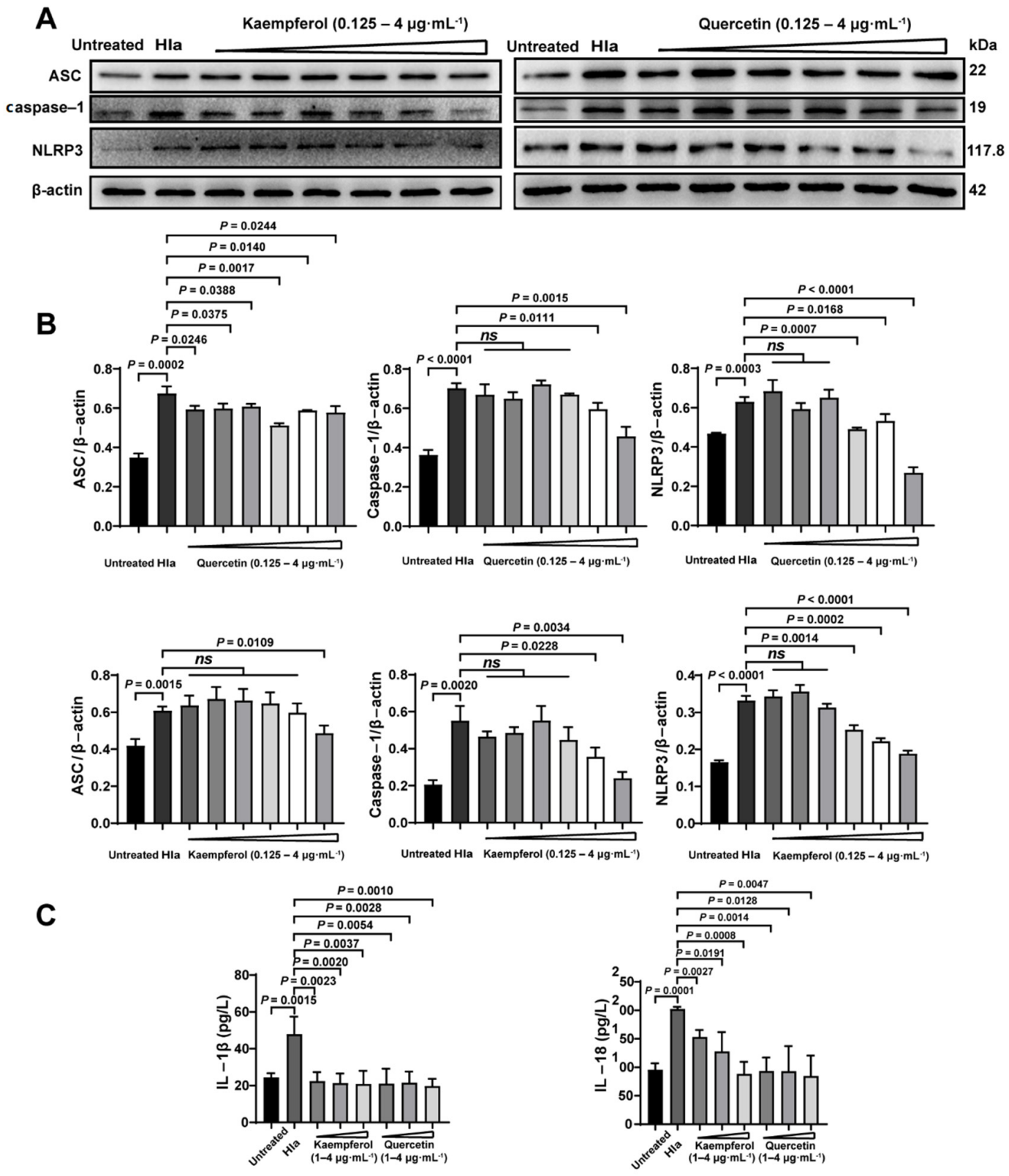

Figure 6. Flavonoids decreased the inflammatory levels caused by Hla in vitro detection: Pulmonary microvascular endothelial cells (PMVECs) were treated Hla with $4 \mathrm{~h}$ (A) ASC, Caspase-1 and NLRP3 expressions of Kaempferol and quercetin treatment were detected by Western Blot. (B) The corresponding proteins analyzed from A were normalized to the of levels of $\beta$-actin. (C) The IL-1 $\beta$ and IL-18 levels were determined by ELISA assay. 


\section{Discussion}

Flavonoid compounds contain oxygen heterocyclic ring and its basic skeleton is 2-phenylchromone. They are widely found in various Chinese herbal medicines and have good antibacterial and anti-inflammatory activities. Most flavonoids, such as luteolin, kaempferol, licochalcone A, apigenin, quercetin, and catechins, have been reported as having an antibacterial effect $[23,24]$. This role is currently one of the important directions for research and development of new antibacterial drugs and new food additives [25]. Baicalin is a traditional Chinese medicine component of flavonoids extracted from Scutellaria baicalensis Georgi. In clinical studies, it has been found to have multiple biological activities, namely, anti-tumor [26], anti-oxidation [27], anti-virus [28], antibacterial, and anti-inflammatory [29]. Among them, in addition to direct antibacterial aspects, studies have shown that baicalin can produce synergistic antibacterial activity against Staphylococcus aureus by inhibiting NorA efflux protein with oxacillin, tetracycline, and ciprofloxacin $[15,30]$. Reports show that antibacterial activity of catechins isolated from cashew nut shells and found that catechins have the potential to resist methicillin-resistant Staphylococcus aureus (MRSA), which can increase the production of reactive oxygen species and reduce the oxidative stress of antioxidant enzymes [31]. Kaempferol is found in many natural plants such as Bupleurum, Eucommia, Hippophae rhamnoides, Ginkgo biloba, Panax notoginseng, mulberry branches, and acacia flowers. Kaempferol 3-rutanoside isolated from acacia flowers can inhibit the adhesion and invasion of Streptococcus mutans to the host by inhibiting the activity of sortase A and exert antibacterial effects [32]. The antibacterial activity of different flavonoids was related to lipophilicity. Reports showed that kaempferol can cause damage to bacterial cells by interacting with the polar head group of the model cell membrane [33,34]. Quercetin is mainly distributed in loquat, rhodiola, raspberry, chrysanthemum, sweet dices, and forsythia. In the study of nine flavonoids from the leaves of Scutellaria baicalensis Georgi, it was found that quercetin-3-glucoside can inhibit Staphylococcus aureus and reduce biofilm formation [35]. In addition, it was found in another study that the combination of quercetin and amoxicillin has synergistic antibacterial activity against amoxicillin-resistant Staphylococcus epidermidis [36]. When observing the inhibitory effect of quercetin on oral microorganisms against Gram-negative bacteria, it was found that quercetin had strong antibacterial activity against Porphyromonas gingivalis, and its minimum inhibitory concentration is $0.0125 \mu \mathrm{g} / \mathrm{mL}$ [37]. In this article, we measured the minimum inhibitory concentration of four flavonoids in traditional Chinese medicine and found that the MICs of baicalin and catechin against Staphylococcus aureus were $128 \mu \mathrm{g} / \mathrm{mL}$ and $1024 \mu \mathrm{g} / \mathrm{mL}$, respectively. The MIC of kaempferol and quercetin was greater than $2048 \mu \mathrm{g} / \mathrm{mL}$. However, in the bacterial growth heat map, we found that kaempferol and quercetin could better inhibit the growth of $S$. aureus in a concentrationdependent manner in the early stage of co-culture and have a certain antibacterial effect.

Current studies have found that Hla can enhance the proliferation and spread of Gram-negative bacteria by preventing acidification of phagosomes of bacterial-containing macrophages, which is a key factor in causing mixed bacterial infections [11]. Therefore, on the basis of the previous research, we explored whether the flavonoids with better inhibitory effect on Hla could inhibit the coinfection of S. aureus and P. aeruginosa. First, the Punnett square was used to observe the direct inhibitory effect of the flavonoids on the mixed culture in vitro. The results showed that baicalin, catechin, kaempferol, and quercetin had better inhibitory effects on mixed culture. Of note, we found that kaempferol showed stronger antibacterial activity when the ratio of $S$. aureus in the mixed culture decreased, suggesting that kaempferol has a strong antibacterial activity against $P$. aeruginosa. Moreover, when counting the number of lung colony in the coinfection mice model, it was found that both kaempferol treatment and prevention group could reduce the number of $P$. aeruginosa in lung tissue, indicating that kaempferol could effectively inhibit the growth of $P$. aeruginosa in vivo and in vitro. Mourouge et al. found that Kaempferol-3o- $\left(2^{\prime}, 6^{\prime}\right.$-di-o-trans-p-coumaryl)- $\beta$-d-glucopyranoside (KF) isolated from Melastomataceae, which can inhibit $P$. aeruginosa by inhibiting the expression of virulence factors, affects 
its nutrient absorption, movement, and growth mechanism when they studied the use of plants to treat acute $P$. aeruginosa infections, indicating that kaempferol has great potential in the inhibition of P. aeruginosa [38].

A mice model of bacterial coinfection was established by tail vein injection. The weight changes in mice before and after infection were counted, and lung tissue colony counts, lung pathological sections, and serum inflammatory factor levels were tested to observe the success of the coinfection model and evaluate the protective effects of kaempferol and quercetin on mixed infected mice. The results showed that the coinfection group caused higher body weight changes than the single infection group, it also promoted the growth of $S$. aureus and P. aeruginosa in the lungs, causing more inflammation than a single infection. This is consistent with the results of a previous Cohen study that $S$. aureus can enhance the proliferation and lethality of Gram-negative bacteria [11]. This showed that the inflammation model of coinfection was successfully established. In the use of flavonoids for prevention and treatment, we found that kaempferol and quercetin, which have good inhibitory effect on Hla, can reduce the weight change in mice before and after infection, it can reduce the accumulation of $P$. aeruginosa in lung tissue and lung injury, and inhibit the expression of inflammatory factors, which has a protective effect on coinfection mice. These results indicate that flavonoids play a promising role in combating bacterial coinfection and they also provide a reference for the development and utilization of plant extracts rich in flavonoids in the future.

Author Contributions: H.D. conceived the project. X.L. and H.D. performed the research design. S.H., Q.D., B.L., F.Y., X.Y. and D.G. performed the experiments. X.L., S.H., Q.D., B.L., F.Y., X.Y. and D.G. performed data analysis. X.L. wrote the manuscript. All authors have read and agreed to the published version of the manuscript.

Funding: This research was funded by [Natural Science Foundation of Beijing, China] grant number [2033202064-21].

Institutional Review Board Statement: The study was conducted according to the guidelines of the Declaration of Helsinki, and approved by Beijing university of agriculture (SYXK, 2015-0004).

Informed Consent Statement: Not applicable.

Data Availability Statement: Not applicable.

Conflicts of Interest: All authors declare no conflict of interest.

Sample Availability: Not applicable.

\section{References}

1. Liu, X.; Liu, F.; Ding, S.; Shen, J.; Zhu, K. Sublethal levels of antibiotics promote bacterial persistence in epithelial cells. Adv. Sci. 2020, 7, 1900840. [CrossRef] [PubMed]

2. Qiu, J.; Nie, Y.; Zhao, Y.; Zhang, Y.; Li, L.; Wang, R.; Wang, M.; Chen, S.; Wang, J.; Li, Y.Q.; et al. Safeguarding intestine cells against enteropathogenic Escherichia coli by intracellular protein reaction, a preventive antibacterial mechanism. Proc. Natl. Acad. Sci. USA 2020, 117, 5260-5268. [CrossRef] [PubMed]

3. Rowe, S.E.; Wagner, N.J.; Li, L.; Beam, J.E.; Wilkinson, A.D.; Radlinski, L.C.; Zhang, Q.; Miao, E.A.; Conlon, B.P. Reactive oxygen species induce antibiotic tolerance during systemic Staphylococcus aureus infection. Nat. Microbiol. 2020, 5, 282-290. [CrossRef]

4. Rivera-Chavez, F.; Zhang, L.F.; Faber, F.; Lopez, C.A.; Byndloss, M.X.; Olsan, E.E.; Xu, G.; Velazquez, E.M.; Lebrilla, C.B.; Winter, S.E.; et al. Depletion of butyrate-producing Clostridia from the gut microbiota drives an aerobic luminal expansion of Salmonella. Cell Host Microbe 2016, 19, 443-454. [CrossRef] [PubMed]

5. Bakkeren, E.; Huisman, J.S.; Fattinger, S.A.; Hausmann, A.; Furter, M.; Egli, A.; Slack, E.; Sellin, M.E.; Bonhoeffer, S.; Regoes, R.R.; et al. Salmonella persisters promote the spread of antibiotic resistance plasmids in the gut. Nature 2019, 573, 276-280. [CrossRef] [PubMed]

6. Barnabie, P.M.; Whiteley, M. Iron-mediated control of Pseudomonas aeruginosa-Staphylococcus aureus interactions in the cystic fibrosis lung. J. Bacteriol. 2015, 197, 2250-2251. [CrossRef]

7. Baldan, R.; Cigana, C.; Testa, F.; Bianconi, I.; De Simone, M.; Pellin, D.; Di Serio, C.; Bragonzi, A.; Cirillo, D.M. Adaptation of Pseudomonas aeruginosa in cystic fibrosis airways influences virulence of Staphylococcus aureus in vitro and murine models of co-infection. PLoS ONE 2014, 9, e89614. [CrossRef] [PubMed] 
8. Maliniak, M.L.; Stecenko, A.A.; McCarty, N.A. A longitudinal analysis of chronic MRSA and Pseudomonas aeruginosa co-infection in cystic fibrosis: A single-center study. J. Cyst. Fibros. 2016, 15, 350-356. [CrossRef]

9. Stacy, A.; Andrade-Oliveira, V.; McCulloch, J.A.; Hild, B.; Oh, J.H.; Perez-Chaparro, P.J.; Sim, C.K.; Lim, A.I.; Link, V.M.; Enamorado, M.; et al. Infection trains the host for microbiota-enhanced resistance to pathogens. Cell 2021, 184, 615-627. [CrossRef]

10. Wu, Y.; Wang, Y.; Yang, H.; Li, Q.; Gong, X.; Zhang, G.; Zhu, K. Resident bacteria contribute to opportunistic infections of the respiratory tract. PLoS Pathog. 2021, 17, e1009436. [CrossRef] [PubMed]

11. Cohen, T.S.; Hilliard, J.J.; Jones-Nelson, O.; Keller, A.E.; O’Day, T.; Tkaczyk, C.; DiGiandomenico, A.; Hamilton, M.; Pelletier, M.; Wang, Q.; et al. Staphylococcus aureus $\alpha$ toxin potentiates opportunistic bacterial lung infections. Sci. Transl. Med. 2016, 8, 329ra31. [CrossRef]

12. Seilie, E.S.; Bubeck Wardenburg, J. Staphylococcus aureus pore-forming toxins: The interface of pathogen and host complexity. Semin. Cell Dev. Biol. 2017, 72, 101-116. [CrossRef] [PubMed]

13. Jordan, P.M.; Gerstmeier, J.; Pace, S.; Bilancia, R.; Rao, Z.; Borner, F.; Miek, L.; Gutierrez-Gutierrez, O.; Arakandy, V.; Rossi, A.; et al. Staphylococcus aureus-derived alpha-hemolysin evokes generation of specialized pro-resolving mediators promoting inflammation resolution. Cell Rep. 2020, 33, 108247. [CrossRef]

14. Li, T.; Zhuang, S.X.; Wang, Y.W.; Wang, Y.L.; Wang, W.H.; Zhang, H.H.; Chen, L.; Wang, D.F.; Zhou, Z.M.; Yang, W.P. Flavonoid profiling of a traditional Chinese medicine formula of Huangqin Tang using high performance liquid chromatography. Acta Pharm. Sin. B 2016, 6, 148-157. [CrossRef] [PubMed]

15. Wang, S.Y.; Sun, Z.L.; Liu, T.; Gibbons, S.; Zhang, W.J.; Qing, M. Flavonoids from Sophora moorcroftiana and their synergistic antibacterial effects on MRSA. Phytother. Res. 2014, 28, 1071-1076. [CrossRef]

16. Song, M.; Liu, Y.; Li, T.; Liu, X.; Hao, Z.; Ding, S.; Panichayupakaranant, P.; Zhu, K.; Shen, J. Plant natural flavonoids against multidrug resistant pathogens. Adv. Sci. 2021, 8, e2100749. [CrossRef] [PubMed]

17. Liu, X.; Zhang, Y.; Li, Z.; Zhang, P.; Sun, Y.J.; Wu, Y.J. Paeoniflorin derivative in paeoniae radix aqueous extract suppresses alpha-toxin of Staphylococcus aureus. Front. Microbiol. 2021, 12, 649390. [CrossRef] [PubMed]

18. Liu, X.; Dong, H.; Wang, M.; Gao, Y.; Zhang, T.; Hu, G.; Duan, H.; Mu, X. IL-1alpha-induced microvascular endothelial cells promote neutrophil killing by increasing MMP-9 concentration and lysozyme activity. Immunol. Res. 2016, 64, 133-142. [CrossRef] [PubMed]

19. Liu, X.; Zhang, H.; He, S.; Mu, X.; Hu, G.; Dong, H. Endothelial-derived interleukin-1alpha activates innate immunity by promoting the bactericidal activity of transendothelial neutrophils. Front. Cell Dev. Biol. 2020, 8, 590. [CrossRef] [PubMed]

20. Lewis, K. The science of antibiotic discovery. Cell 2020, 181, 29-45. [CrossRef]

21. Meier, D.; Hernández, M.V.; van Geelen, L.; Muharini, R.; Proksch, P.; Bandow, J.E.; Kalscheuer, R. The plant-derived chalcone Xanthoangelol targets the membrane of Gram-positive bacteria. Bioorg. Med. Chem. 2019, 27, 115151. [CrossRef]

22. Qiu, J.Z.; Niu, X.D.; Dong, J.; Wang, D.; Wang, J.; Li, H.; Luo, M.; Li, S.; Feng, H.; Deng, X. Baicalin protects mice from Staphylococcus aureus pneumonia via inhibition of the cytolytic activity of alpha-hemolysin. J. Infect. Dis. 2012, 206, 292-301. [CrossRef] [PubMed]

23. Yike, Z.; Di, W.; Xiaodan, H.; Tong, Z.; Yongxia, W. Progress in the study of antibacterial effect of flavonoids compounds. China Food Addit. 2019, 30, 166-171.

24. Yeon, K.A.; Xuejiao, S.; Chengcheng, H.; Lei, S.; Xiang, G.; Chunlian, T.; Mingchun, L. Mechanism of flavonoids on drug resistance inhibition resistance. Anim. Husb. Vet. Med. 2016, 48, 117-120.

25. Caixia, Y.; Chunlian, T.; Jian, G.; Xue, H.; Limei, H.; Mingchun, L. Research progress of antibacterial action and mechanism of flavonoids. China Anim. Husb. Vet. Med. 2014, 41, 158-162.

26. Baiyan, W.; Shuxian, L.; Qianqian, H.; Ruiqin, L. Advances in research on anti-tumor effects of baicalin. Mod. Med. J. China 2020, 22, 101-103.

27. Wei, X.; Zhu, X.; Hu, N.; Zhang, X.; Sun, T.; Xu, J.; Bian, X. Baicalin attenuates angiotensin II-induced endothelial dysfunction. Biochem. Biophys. Res. Commun. 2015, 465, 101-107. [CrossRef] [PubMed]

28. Weiwei, L.; Kaijun, J.; Zhongbai, S.; Liangong, C.; Shuhao, L.; Youxian, W.; Jinyang, S. Research progress on antiviral pharmacological effects of baocalin. J. Xuzhou Inst. Technol. (Nat. Sci. Ed.) 2020, 35, 13-17.

29. Hao, L.; Zibing, Z.; Xin, W. Research progess on antibacterial activity of baicalin. Anim. Husb. Feed. Sci. 2017, $38,61-63$.

30. Qiu, F.; Meng, L.; Chen, J.; Jin, H.; Jiang, L. In vitro activity of five flavones from Scutellaria baicalensis in combination with cefazolin against methicillin resistant Staphylococcus aureus (MRSA). Med. Chem. Res. 2016, 25, 2214-2219. [CrossRef]

31. Simran, S.; Vellingiri, V. Catechin isolated from cashew nut shell exhibits antibacterial activity against clinical isolates of MRSA through ROS-mediated oxidative stress. Appl. Microbiol. Biotechnol. 2020, 104, 8279-8297.

32. Yang, W.Y.; Won, T.H.; Ahn, C.H.; Lee, S.H.; Yang, H.C.; Shin, J.; Oh, K.B. Streptococcus mutans sortase a inhibitory metabolites from the flowers of Sophora japonica. Bioorg. Med. Chem. Lett. 2015, 25, 1394-1397. [CrossRef]

33. Echeverria, J.; Opazo, J.; Mendoza, L.; Urzua, A.; Wilkens, M. Structure-activity and lipophilicity relationships of selected antibacterial natural flavones and flavanones of chilean flora. Molecules 2017, 22, 608. [CrossRef] [PubMed]

34. He, M.Y.; Wu, T.; Pan, S.Y.; Xu, X.Y. Antimicrobial mechanism of flavonoids against Escherichia coli ATCC 25922 by model membrane study. Appl. Surf. Sci. 2014, 305, 515-521. [CrossRef]

35. Narendran, R.; Shankar, S.; Rene, C.L.; Sundaram, M.M.; Sai, S.N.; Brindha, P.; Aravind, S. Antimicrobial flavonoids isolated from Indian medicinal plant Scutellaria oblonga inhibit biofilms formed by common food pathogens. Nat. Prod. Res. 2016, 30, $2002-2006$. 
36. Siriwong, S.; Teethaisong, Y.; Thumanu, K.; Dunkhunthod, B.; Eumkeb, G. The synergy and mode of action of quercetin plus amoxicillin against amoxicillin-resistant Staphylococcus epidermidis. BMC Pharmacol. Toxicol. 2016, 17, 39. [CrossRef]

37. Geoghegan, F.; Wong, R.W.; Rabie, A.B. Inhibitory effect of quercetin on periodontal pathogens in vitro. Phytother. Res. 2010, 24, 817-820. [CrossRef]

38. Alwash, M.S.; Aqma, W.S.; Ahmad, W.Y.; Ibrahim, N. Transcriptomic response in Pseudomonas aeruginosa towards treatment with a kaempferol isolated from melastoma malabathricum linn Leaves. Int. J. Microbiol. 2020, 2020, 6915483. [CrossRef] [PubMed] 